Wilfrid Laurier University

Scholars Commons @ Laurier

Physics and Computer Science Faculty

Publications

Physics and Computer Science

1989

\title{
Driven Pendulum for Studying Chaos
}

James A. Blackburn

Wilfrid Laurier University, jabjabjab@cogeco.ca

S. Vik

Wilfrid Laurier University

Binruo Wu

Wilfrid Laurier University

H.J.T. Smith

University of Waterloo

Follow this and additional works at: https://scholars.wlu.ca/phys_faculty

\section{Recommended Citation}

Blackburn, James A.; Vik, S.; Wu, Binruo; and Smith, H.J.T., "Driven Pendulum for Studying Chaos" (1989). Physics and Computer Science Faculty Publications. 34.

https://scholars.wlu.ca/phys_faculty/34

This Article is brought to you for free and open access by the Physics and Computer Science at Scholars Commons @ Laurier. It has been accepted for inclusion in Physics and Computer Science Faculty Publications by an authorized administrator of Scholars Commons @ Laurier. For more information, please contact scholarscommons@wlu.ca. 


\title{
Driven pendulum for studying chaos
}

\author{
James A. Blackburn, S. Vik, and Binruo Wu ${ }^{2)}$ \\ Department of Physics and Computing, Wilfrid Laurier University, Waterloo, Ontario, Canada
}

H. J.T. Smith

Department of Physics, University of Waterloo, Waterloo, Ontario, Canada

(Received 23 May 1988; accepted for publication 10 November 1988)

\begin{abstract}
An instrument suitable for experimental studies of chaotic motion is described. It consists of a mechanical pendulum driven by both steady and alternating torques, together with interface electronics for data logging. An optical shaft encoder serves as the transducer for measuring the instantaneous pendulum coordinate. The entire system is controlled from a personal computer. Sample phase-plane and Poincaré plots are presented.
\end{abstract}

\section{INTRODUCTION}

Chaos, which is a type of essentially unpredictable behavior exhibited by certain nonlinear deterministic systems, has been a subject of intense interest during recent years. The overwhelming majority of publications in this field either have been highly mathematical in nature or have attacked the relevant equations through the methods of numerical analysis. The former approach can sometimes obscure the physical nature of the phenomenon under investigation, while the latter (often, unfortunately) can consume prodigious amounts of computer time. For these and other reasons, it is highly desirable to carry out direct experimental studies of representative physical systems.

Chaotic behavior has been observed in periodically forced neural oscillators, ${ }^{3}$ in driven acoustic systems, ${ }^{2}$ in driven piezoelectric resonators, ${ }^{3}$ in ac-driven diode circuits ${ }^{4}$ and photoconductors, ${ }^{5}$ in electron-hole plasmas in Ge crystals, ${ }^{6}$ in resonantly forced surface water waves, ${ }^{7}$ and in irradiated superconducting Josephson junctions. ${ }^{8}$ This is an instructive, but by no means complete, indication of the variety of experimental work which has already been done. What is typical, however, is that each such study presents only a partial elaboration of the connection between the observed chaotic aspects and the relevant theoretical model.

A driven damped pendulum can serve as an excellent experimental test bed for studying chaos, in part because its description is essentially complete, more so since its motion can be followed in detail. It also, incidentally, can serve as a mechanical analog of a capacitive Josephson junction. This correspondence led Sobolewski et al. ${ }^{9}$ to remark that Josephson junctions "are an ideal experimental tool to test the theoretical predictions of the driven damped pendulum model." However, the fact that Josephson oscillations typically occur at frequencies about $10^{12}$ times higher than those in a pendulum means that it is not feasible to actually observe detailed junction dynamics. What can be measured are the dc current-voltage characteristics, but as these authors point out, "the experimental curve represents an average over an enormous number of switching events." In other words, only a blurred time average of the underlying phenomenon is seen. For these reasons we would suggest instead that it is the driven damped pendulum itself which represents the ideal experimental system.
An essential element in any pendulum experiment is a suitable transducer for sensing the instantaneous angular coordinate $(\theta)$. In a previous work, ${ }^{10}$ a device known as a resolver was employed for this purpose. Although the overall performance was good, it was felt that significant improvements could be made, and a complete redesign of the apparatus was undertaken. This paper describes the new system. An optical shaft encoder is now used for determining $\theta$; this choice resulted in reduced friction and inertia. In addition, the pendulum itself was made more compact, thus raising its natural frequency by a factor of 3 and comparably reducing the experimental run times. Eddy current damping is now adjustable. Finally, new interface electronics were required by the switch to an optical encoder. Under computer control, both de and ac torques may be applied and the resulting motion recorded with considerable precision. In the concluding section some sample observations are presented to demonstrate the versatility and accuracy of the system.

\section{APPARATUS}

The equation of motion of a driven damped pendulum is

$$
\ddot{I}+\dot{\theta} \dot{\theta}+(m g r) \sin (\theta)=\tau_{0}+\tau_{1} \sin (\omega t),
$$

where $\theta$ is the angular coordinate of the pendulum mass $m$, which is suspended a distance $r$ below the pivot point, $b$ is a damping coefficient, $I$ is the total moment of inertia of all rotating components, $\tau_{0}$ is a constant applied torque, and $\left(\tau_{1}, \omega\right)$ are the amplitude and frequency, respectively, of the harmonic torque component. The system is characterized by its undamped natural frequency $\left.\omega_{0}=\sqrt{(m g r /} / \bar{I}\right)$ and critical torque (mgr).

An exploded view of the apparatus is shown in Fig. 1. All components which undergo rotational motion were either coaxially attached to, or suspended from, a horizontally aligned hardened steel shaft which was supported at its ends by ball races set in a rigid frame. To this axle were mounted in turn the pendulum mass, an optical shaft encoder, and an annular-ring permanent magnet. The magnet formed the rotor of a brushless, slotless, linear motor whose stator drive coils were fixed to the nonmoving frame. Finally, a solid copper eddy-current damping plate was also attached to the apparatus frame-in such a way that its linear separation from the rotating ring magnet was adjustable by means of a micrometer screw. 


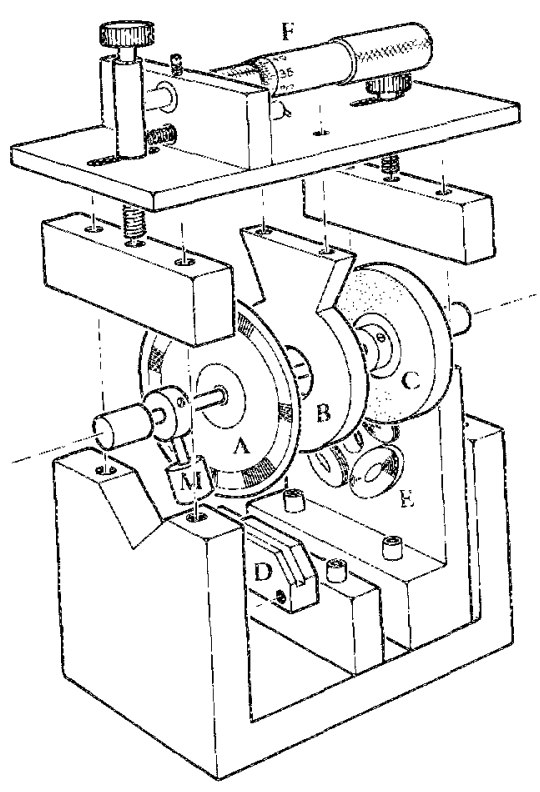

Frg. 1. Exploded view of the damped, driven pendulum. Approximate overall dimensions are $5 \mathrm{~cm} \times 9 \mathrm{~cm} \times 9 \mathrm{~cm}$. Labeled components are $M$, pendulum mass; $A$, code wheel; $B$, copper damping plate; $C$, ring magnet; $D$, encoder module; $\mathrm{E}$, motor coils; and $\mathrm{F}$, micrometer.

The pendulum itself consisted of a short cylinder of aluminum or brass threaded onto a small connecting rod whose other end was screwed into a mounting collar on the steel shaft (the pendulum motion was thus constrained to a vertical plane).

As noted in the Introduction, the anguiar displacement of the pendulum with respect to a vertical reference line was sensed by an optical shaft encoder comprised of a Hewlet:Packard model HEDS-9000 encoder module and a Hewlet $\hat{\text { - }}$ Packard model HEDS-6100 code wheel. The code wheel is a 5 -cm-diam metal foil disk with 1000 very fine slots equally spaced around its periphery. The encoder is a small encapsulated unit containing a lensed light-emitting diode source on one side of a 70-mil gap and a photodiode array with signalprocessing circuitry on the other side; it is mounted so that the edge of the code wheel extends into the gap. As the code wheel rotates, the light signal passing between the emitter and photodiodes is interrupted by the moving siots. The HEDS-9000 output consists of TTL compatible square waves from two channels. Because channel A is (ideally) 90 electrical degrees out of phase with channel $B$, the interval between successive slot transits is marked by four distinct $A$ / $B$ patterns. Thus a code wheel with 1000 slots can generate raw data with a resolution of one part in 4000 , that is, a precision of approximately 12 bits.

The means of delivering torque to the pendulum was similar to one employed in an earlier design. ${ }^{10}$ As before, a brushless, slotless, linear motor was disassembled and only the ring-magnet rotor and drive coils were retained. Previousiy, the coils were left on the original motor backing plate, but it became apparent that some undesirable electrodynamic damping effects were generated by the proximity of this metal plate to both the coils and the magnetic rotor, and so in the present system the coils were remounted on a phe-

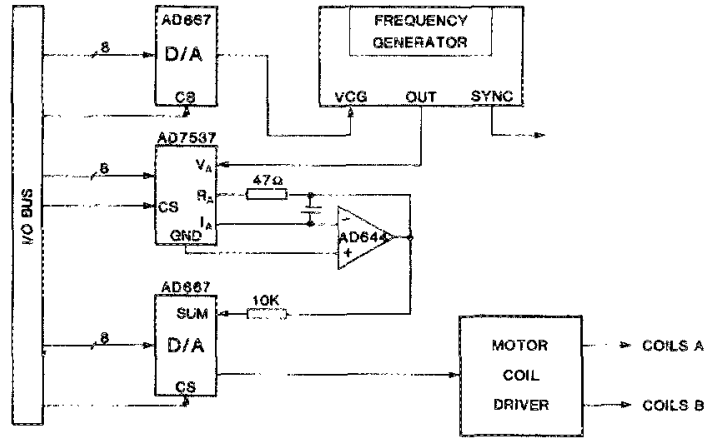

Fic. 2. Control circuit for delivering external torgue to the pendulum. Communication with the computer is via the $1 / 0$ bus. Wher energized, the stator coils induce a torque on the ring-magnet rotor.

nolic support. Damping was therefore provided exclusively by the copper plate which is coplanar with the ring magnet.

\section{SYSTEM CONTPOL AND DATA ACQUISITION}

Experimental control and data acquisition were performed by a personal computer which was equipped with a JDR Microdevices PR-2 interface card. "This provided a convenient and easy-to-use data and command bus for communicating within the system environment. The $\mathrm{PC}$ was a generic turbo XT containing a NEC V20 CPU chip operating at $10 \mathrm{MHz}$ and an 8087 math coprocessor. An IBM VGA adapter and monitor allowed high-resolution realtime color plots to be generated.

\section{A. Torque}

A prime requirement of the hardware/software was the ability to independently vary two fundamental parameters of the pendulum, namely, the amplitude and frequency of the applied torque. As shown in Fig. 2, the sine wave oscillator output is first passed through a 12-bit multiplying digital-toanalog converter (D/A) (AD 7537). The output voltage from the $\mathrm{AD} 644$ op-amp is $-(K / 4096) V_{A}$, where $K$ is a 12-bit number previously sent as two successive bytes to the AD 7537 from the PC. The multiplier is thus functioning as a digitally controlled attenuator which provides torque amplitude control. The AD 667 converter adds dc level shifting to the ac drive signal by means of its summing capability. This chip was pin programmed in the bipolar $+/-5-\mathrm{V}$ mode and was loaded in a two-byte sequence with a 12-bit number $L$. Its output was the inverted multiplier output ac voltage plus the dc value $(L / 2048-1) 5 \mathrm{~V}$. The resulting torque, which was directly proportional to the coil amplifier signal, therefore had both programmable ac amplitude and de components.

The Wavetek model $182 \mathrm{~A}$ signal generator permitted external frequency adjustment through a VCG input on its front panel. The sensitivity of this input was such that if, for example, the signal generator was set at $1 \mathrm{~Hz}$, then the control factor was $1 \mathrm{~Hz}$ per volt. As shown in Fig. 2, computer selection of the oscillator frequency was effected by means of the programmable voltage produced by a second 12 -bit $\mathrm{D} / \mathrm{A}$ converter. This, in turn, directly determined the frequency of the torque applied to the pendulum. 


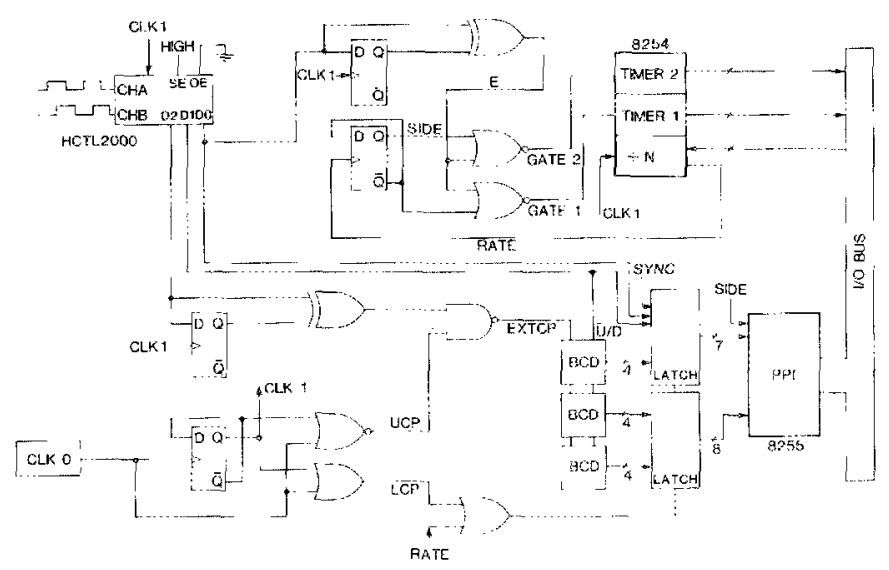

Fici. 3. Data-acquisition interface circuit. Quadrature signals from the optical code wheel enter the HCTL-2000 decoder/counter. The final angle and timing data are transferred to the computer on the $1 / 0$ bus.

\section{B. Motion}

Once the desired torque is turned on and the pendulum is undergoing its periodic or chaotic motion, it is necessary to acquire positional data from the angular transducer. For presentation in the form of a phase-plane plot, the final data must be in the form of a rapidly sampled time series $\theta(t)$ together with matching angular velocities $\theta(t)$, whereas a Poincaré plot requires $\theta$ and $\dot{\theta}$ to be evaluated only once in each cycle of the ac torque. The capability to perform data acquisition in either format is provided by the circuit shown in Fig. 3. Before proceeding with a detailed analysis of the logic, the principal features of its operation may be noted: (1) Pulses derived from the rotating code wheel are accumulated in a modulo 4000 up/down counter. (2) A periodic waveform (SIDE) serves as the sampling time base. (3) The contents of the angle counter, and the state of the SYNC pulse from the signal generator, are transferred to latehes just prior to each transition of SIDE. (4) The latches are read by the PC immediately after each transition of SIDE. (5) A timer is used to determine the elapsed interval since the last code wheel pulse. (6) This timing information is read by the $\mathrm{PC}$ in conjunction with the latch data and is used to compute the angular velocity of the pendulum.

\section{T. Angle}

The quadrature square wave signals from the encoder module are processed by a Hewiett-Packard HCTL-2000 decoder/counter interface integrated circuit. This special purpose chip contains a digital filter, quadrature decoding logic, a 12-bit binary counter, a 12-bit latch, a 16-to-8 line multiplexer, and an 8-bit tristate output buffer. As noted earlier, the fact that the code wheel has 1000 slots means that a complete revolution generates 4000 quadrature events. This number does not match the 4096 states of a 12-bit binary code, and thus separate counters external to the HCTL2000 must be employed in order for the hardware to autonomously track multiple flips of the pendulum. By wiring $\mathrm{OE}$ low and SEL high, the HCTL-2000 is placed in the low byte read mode; this is done since only the three lowest-order bits $\left(D_{0}, D_{1}, D_{2}\right)$ will be used.

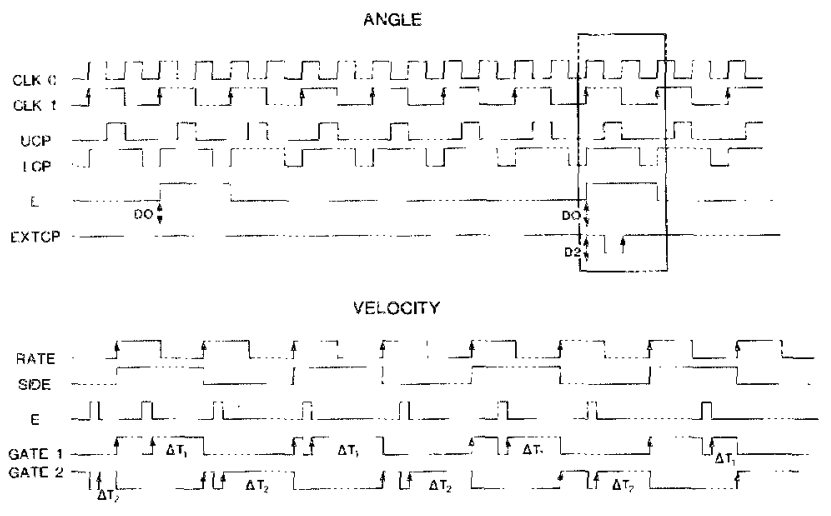

FiG. 4. Timing diagram for the principal signals in the interface circuit. The apper group are referenced to CL.KO, while the lower waveforms are sychronized to RATE, which runs at the user-selested sampling frequency. The framed portion highlights the appearance of a pulse on EXTCP following a state change in bit $D_{2}$.

A timing diagram is illustrated in Fig. 4. The system clock $(\mathrm{CLKO}=500 \mathrm{kHz})$ is divided down to $\mathrm{CLK} 1=250$ $\mathrm{kHz}$ and passed to a number of points in the circuit, including the decoder/counter interface chip. State changes in the HCTL-2000) occur on rising edges of CLK1. The short (1$\mu$ s) pulse which appears on EXTCP each time bit $D_{2}$ changes either from 1 to 0 or from 0 to 1 serves as an external clock for three cascaded binary coded decimal (BCD) up/ down counters (74190). It is not difficult to show that if $D_{1}$ is 0 following a change (to either state) of $D_{2}$, then an upcount is in progress; hence $D_{1}$ directly controls the $\bar{U} / D$ mode of the BCD counters. A modulo 4000 counter is thus realized by combining the two lowest bits from the HCTL2000 with the 12 bits from the BCD counters.

The Intel 8254 Programmable Interval Timer contains three independent 16-bit counters. One of these was set via the input-output (I/O) bus to mode 3 and loaded under PC control with an integer $N$. The output of this counter (RATE) is a square wave whose period is $N$ times that of CLK1. For $N$ even, the duty cycle is $50 \%$, whereas for $N$ odd the output is high during the first $(N+1) / 2$ periods of CLK1 and low for the remaining $(N-1) / 2$. As can be seen from the circuit diagram, the penduium angle, which is being sampled once during every cycle of CLK 1 by the HCTL2000, will be transferred into the latches only when RATE and LCP are both low. This, in fact, occurs $N / 2$ or $(N-1)$ / 2 times while RATE is low, depending on whether $N$ is even or odd. Since the computer was programmed to accept data immediately following either rising or falling edges of SIDE, only the most recent angle latched prior to each rising edge of RATE is transferred to the PC (via an Intel 8255 Programmable Peripheral Interface chip which is preset to mode 0 ). The value of $N$ could range from 2 to 65535 (that is $\left.2^{16}-1\right)$ with corresponding sampling rates from $125 \mathrm{kHz}$ to $3.81 \mathrm{~Hz}$.

\section{Angular velocity}

The two remaining timers in the Intel 8254 are set to mode 2 and count down from a preset value $M$, which in the present case was 65535 . When GATE to either timer goes high, the corresponding counter is reinitialized to $M$ on the 
next rising edge of the clock and commences down-counting on the following falling edge. A low GATE inhibits further counting. The time interval between rising and falling edges of GATE may then be calculated from the final count value and the clock period.

Signal $E$ is produced by the Exclusive $O R$ of $D_{0}$ and the $Q$ output from a $D$-type flip-flop (7474); it goes high for one period of CLK1 every time the least significant bit $D_{0}$ changes state. Because the pendulum, and therefore also the code wheel, is not rotating with constant angular velocity, the intervals between high pulses on $E$ are not equispaced. A hypothetical case of decreasing angular velocity is represented by the $E$ waveform in the lower group of signais shown in Fig. 4. As is evident from GATE1 and GATE2 in the timing diagram, one timer is active while SIDE is high, while the second timer is active while SIDE is low.

The waveforms and sequences described in the preceding two paragraphs are utilized in the following way. As remarked earlier, the computer takes readings immediately after transitions of SIDE. Consider first a falling edge of SIDE and note the lower group of signals in the timing diagram. During the first half of SIDE $=0$ (while RATE $=1$ ), data corresponding to the most recent pendulum angle, denoted by $A_{1}$, will be latched and may be read by the PC. Following this, the interval $\Delta T_{1}$, which is the time clapsed since the last $E$ pulse, may be read from timer 1 . In a similar fashion the $\mathrm{PC}$ can acquire, on the next rising edge of $\mathrm{SLDE}$, a new angle $A_{2}$ and interval $\Delta T_{2}$. The angular velocity $\dot{\theta}$ is obtained from these values with the expression

$$
\dot{\theta}=\left(A_{2}-A_{1}\right) /\left(\Delta T-\Delta T_{2}+\Delta T_{1}\right),
$$

where $\Delta T$ is the period of RATE. The angle $A_{1}$, which was presumed to be valid at the moment when SIDE went low, may now be "corrected":

$$
\theta=A_{1}+\dot{\theta} * \Delta T_{1} \text {. }
$$

This interpolation effectively increases the resolution beyond the 12 bits associated with the raw data; consequently, corrected angles are represented in a 16-bit format. The accuracy of $\theta$ is obviously dependent on the sampling ratewhich is set by the integer $N$. While rates as high as $10 \mathrm{kHz}$ were possible without loss of information, in most cases it was found that $300 \mathrm{~Hz}(N=833)$ was a good choice.

The procedures described above may be applied in a reasonably obvious manner at each successive transition of SIDE to yield the regularly sampled time series $\theta(t)$. For economy of storage, only the $\theta$ values were retained in $\mathrm{PC}$ memory. Angular velocities were recomputed later from the equispaced $\theta(t)$.

When a Poincare plot is reguired, the computer is programmed to monitor the function generator SYNC signal which has also been latched along with the 14-bit code representing the pendulum angle. In this case only particular data pairs $\left\{A_{i}, \Delta T_{i}\right\}$ and $\left\{A_{i+1}, \Delta T_{i+1}\right\}$ are retained in the $\mathrm{PC}$. The first is coincident with, or immediately follows, a rising edge of SYNC, while the second follows one period of RATE later.

The $E$ pulse stream indicated in the timing diagram is only in some sense "typical." A higher value of hypothetical angular velocity would shorten the interval between these

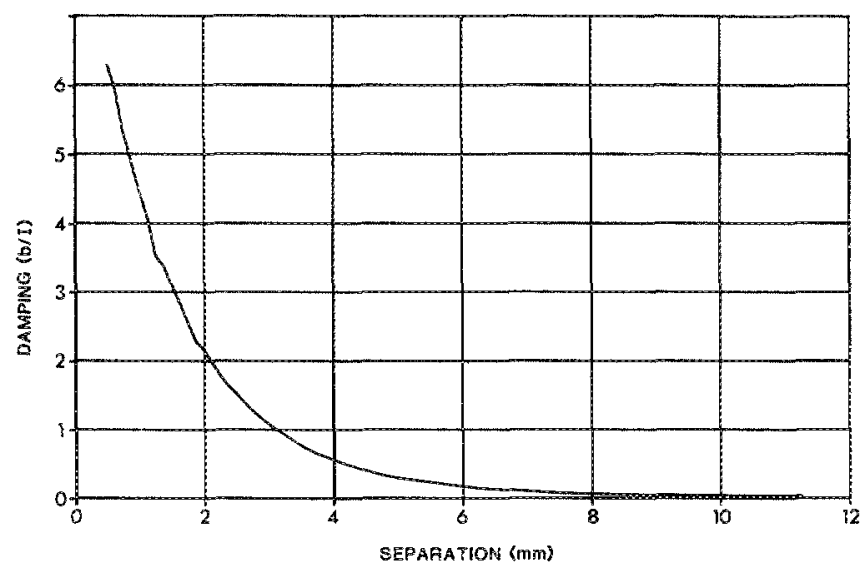

FIG. 5. Dependence of damping coefficient $b / I$ on the separation of the rotor magnet and copper eddy-current plate.

pulses so that severai might occur within some half-cycles of SIDE. Consideration of this situation reveals that valid $\theta$ and $\dot{\theta}$ will still be generated. Slower angular velocities, on the other hand, require special attention by the software since it becomes possible for one or more consecutive half-cycles of SIDE to contain no $E$ pulse.

\section{EXPERIMENTAL RESULTS}

As constructed, the pendulum had an undamped natural frequency $\omega_{0}=2.05 \mathrm{~Hz}$. Eddy-current damping was adjusted by controlling the separation between the stationary copper plate and the rotating ring magnet with a micrometer screw. It was possible to experimentally determine the damping $b / I$ by analyzing the rate of decay of angular velocity when all applied torque was suddenly switched of: For this measurement, the entire pendulum apparatus was temporarily tumed on-end so that rotation was in a horizontal plane and gravitational torque was effectively canceled. The calibration curve shown in Fig. 5 was obtained from a series of such observations carried out at different spacings.

Representative phase-plane and Poincaré plots are illustrated in Figs. 6 and 7, respectively. The former corresponds to multiply periodic motion, while the latter shows a typical

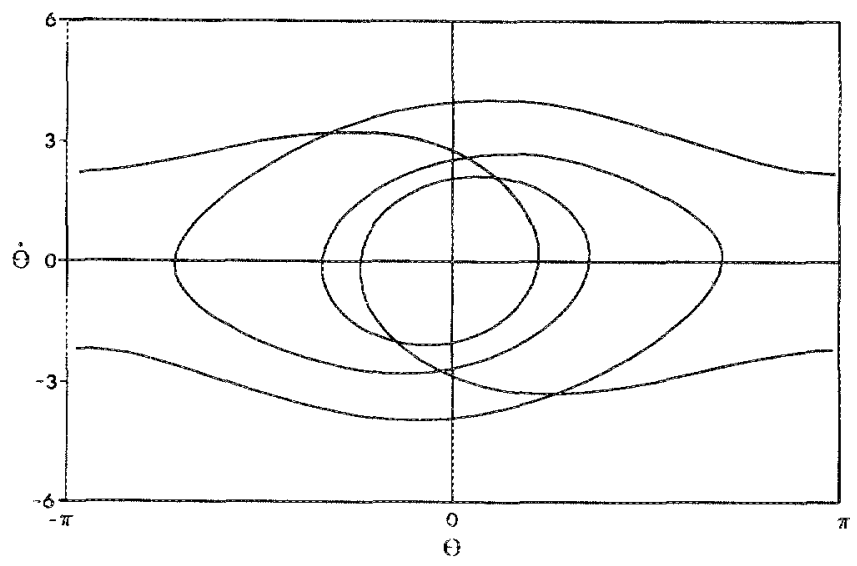

FIG. 6. Typical phase-plane plot obtained from the pendulum. The motion in this case is period 3 . 


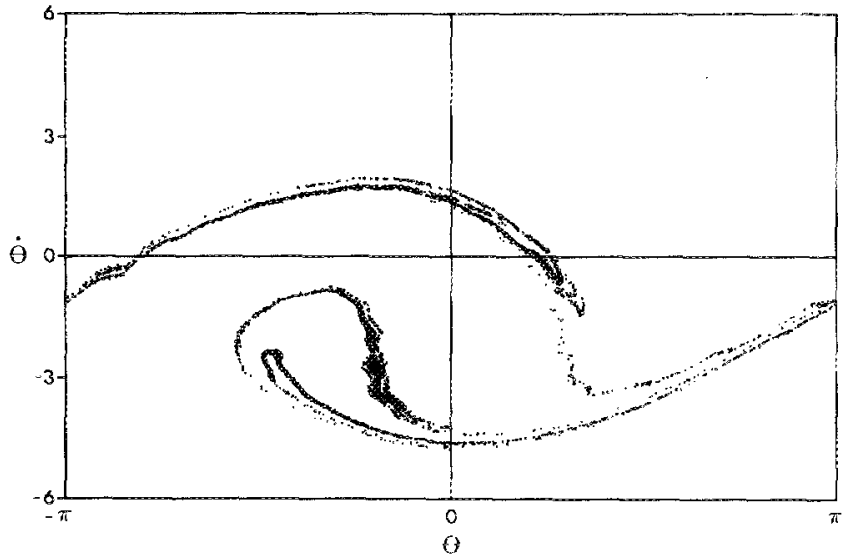

FIG. 7. Poincaré plot. This strange attractor is characteristic of chaotic motion. The figure contains 5000 sampled points.

chaotic strange attractor with its characteristic fractal structure. The precision of the pendulum is clearly evident in these sample figures. The system has in fact proven to be extremely reliable and accurate.

\section{ACKNOWLEDGMENT}

Financial support was provided by the Natural Sciences and Engineering Research Council of Canada.

"Visiting Scholar from Chengdu Institute of Radio Engineering, Chengdu, Sichuan, Pcople's Republic of China.

'K. Aihara, G. Matsumoto, and M. Ichikawa, Phys. Lett. A 111, 251 (1985).

${ }^{2}$ M. Kitano, T. Yabuzaki, and T. Ogawa, Phys. Rev. Lete. 50, 713 (1983). D. J. Jefferies, Phys. Lett. A 90,316 (1982).

'S. 'Tanaka, 'T. Matsumoto, and L. O. Chua, Physica D 28, 317 (1987).

${ }^{5}$ S. W. Tcitsworth and R. M. Westervelt, Phys. Rev. Lett. 56, 516 (1986).

${ }^{6} \mathrm{G}$. A. Held, C. Jefries, and E. E. Haller, Phys. Rev. Lett. 52, 1037 (1984)

${ }^{7}$ M. Funakoshi and S. Inoue, Phys. Lett. A 121, 229 (1987).

"N. F. Peciersen, Phys. Scr. T 13, 129 (1986).

'R. Sobolewski, D. R. Dykaar, T. H. Hsiang, C. Vanneste, and C.-C. Chi, Phys. Rev. B 37, 3778 (1988).

${ }^{10}$ J. A. Blackburn, Yang Zhou-jing, S. Vik, H. 3. T. Smikh, and M. A. H. Nerenberg, Physica D 26, 385 (1987).

"Manufactured by JDR Microdevices, 1224 S. Bascom Avenue, San Jose, CA. 